\title{
Green Synthesis of $\mathrm{NiSnO}_{3} \mathrm{Nanopowder}$ and Its Application as a Hydroquinone Electrochemical Sensor
}

\author{
Taimur Athar ${ }^{*}, \dagger$, Magdy Abdelaal ${ }^{1,2}$, Zeenat Khatoon ${ }^{3, \dagger}$, Amit Kumar $^{3}$, \\ Alabass Razzaq, Aleem Ahmad Khan ${ }^{4}$, Hassan Fouad ${ }^{5,6}$, \\ Shafeeque Ahmed Ansari ${ }^{3}$ and Zubaida Amin Ansari ${ }^{3, * *}$ \\ CSIR-Indian Institute of Chemical Technology, Hyderabad, Andhra Pradesh, 500007, India \\ ${ }^{1}$ Prince Sultan University, College of Engineering, Riyadh, Saudi Arabia \\ ${ }^{2}$ Academy of Civil Aviation, 6th of October Airport, Egypt \\ ${ }^{3}$ Center for Interdisciplinary Research in Basic Sciences, \\ Jamia Millia Islamia, New Delhi, 110025, India \\ ${ }^{4}$ Salar-E-Millat Research Centre and Central Laboratory for Stem Cell Research \\ \& Regenerative Medicine, Centre for Liver Research and Diagnostics, \\ Deccan Medical Sciences, Hyderabad, Telangana 500058, India \\ ${ }^{5}$ Department of Applied Medical Science, Riyadh Community College, King Saud University, \\ Riyadh, 11437 Saudi Arabia \\ ${ }^{6}$ Biomedical Engineering Department, Faculty of Engineering, Helwan University, \\ P.O. Box, 11792, Helwan, Egypt
}

(Received February 13, 2015; accepted May 12, 2015)

Key words: green synthesis, perovskite material, bimetallic oxide, electrochemical sensor, hydroquinone

Green synthesis of $\mathrm{NiSnO}_{3}$ nanopowder was carried out via chemie douce using nickel and tin chlorides as precursors by stirring and refluxing at $80{ }^{\circ} \mathrm{C}$. The dried dark green bimetallic oxide nanoparticle was then used for preparing the sensing electrode in the form of a screen-printed electrode (SPE). The synthesized nanoparticles were analyzed for elemental, structural, and morphological characteristics using various analytical techniques. The Fourier transform infrared (FTIR) spectrum indicated the formation of a strong Sn-O-Ni framework, while X-ray diffraction (XRD) confirmed the formation of a bimetallic oxide nanopowder of $\mathrm{NiSnO}_{3}$. The synthesized nanoparticles were studied for hydroquinone (HQ) sensing characteristics to develop a sensitive and reproducible electrochemical sensor from 1 to $9 \mathrm{mM}$ concentrations in buffer. The developed sensor exhibited a sensitivity of $6.03 \mu \mathrm{A} / \mathrm{mM}\left(46.38 \mu \mathrm{A} / \mathrm{mMcm}^{-2}\right)$. The charge transfer studies indicated a reversible and diffusion-controlled process. To the best of our knowledge, such a material, i.e., $\mathrm{NiSnO}_{3}$, has not be used for HQ sensors and is being reported for the first time.

*Corresponding author: e-mail: taimurathar2001@gmail.com

${ }^{* *}$ Corresponding author: e-mail: zaansari@jmi.ac.in

†Authors contributed equally. 


\section{Introduction}

The investigation of novel materials for device development is very important for technological progress, which requires tailoring of material stoichiometry/composition, crystal size, crystal defects, and surface area with suitable functional properties. ${ }^{(1-6)}$ Most of the metal oxides offer such flexibility with advantages of simple and economic synthesis and possible band gap tailoring. For example, perovskite-type alkaline earth stannates, such as cadmium stannate, are presented as new materials for semiconductor gas sensors because of their high selectivity, sensitivity, and stability towards ethanol gas. (7) These materials are also being explored for their possible application in solar cells and as battery materials. ${ }^{(8-10)}$ With the help of a soft chemical approach, it is easy to convert precursors of single or bimetallic oxides with homogenous size, shape, distribution, and phase purity simply by controlling their aggregation. Therefore, the use of different types of molecular precursor gives unique morphological features that affect both the physical and chemical properties of the final product. Therefore, in this work, a soft-chemical approach was used as a green synthesis technique to synthesize monodispersible and functional Ni-doped tin oxide materials.

Most anthropogenic and economic activities end up introducing chemical contaminants into the natural environment besides producing value-added products for human consumption. Many of these contaminants are fairly toxic and tend to have longterm adverse impact on flora and fauna. These being a source of great concern, there is a continuous need for their detection, detoxification and environmental remediation. Hydroquinone (HQ) is one such environmental contaminant of concern as it is being widely used in pharmaceutical and industrial products. ${ }^{(1)}$ Since it is readily soluble in water, it can pose a threat to human health and hence requires to be detected sensitively using a suitable device. Owing to its unregulated use in cosmetics as a 'skin lightener' and possible long-term adverse impact on human health such as incidence of cancer due to the enhanced exposure of sunlight on treated skin, there is much controversy on its use leading to its total ban in many European countries, the USA, and China, although it has not been classified as a significant carcinogen. However, its continuous topical use or occupational exposure results in the de-pigmentation of skin, which itself can lead to an increased incidence of cancer due to a higher amount of UV irradiation. In view of known health hazards due to occupational exposure and fugitive emissions of HQ, it is necessary to develop a simple and rapid analytical method for the detection of microquantities of HQ. ${ }^{(11,12)}$

The conventional techniques used for the detection and measurement of HQ are based on high-performance liquid chromatography (HPLC), spectrophotometry, chemiluminescence, and electrochemical sensing methods. Among these methods, the electrochemical sensing method has numerous advantages and merits such as reduced costs, simplicity, efficiency, selectivity, and most importantly ease of carrying out analysis without elaborate sampling and pre-procedures such as separation. However, to devise a suitable electrochemical sensing method, it is important to find a suitable matrix that can act as a transducer and mediate the signal generated through chemical reactions between the matrix and the target molecule. There are two important determinants, namely, effective adsorption and large surface area, for the matrix to function 
appropriately. A number of nanomaterials have recently been used as efficient electron mediators for the fabrication of various electrochemical sensors that have since proved both effective as well as reliable. ${ }^{(11-15)}$

Among these nanomaterials, zinc oxide, conducting polymers, graphene and carbon have been used for the electrochemical detection of HQ. For example, da Silva et al. modified a carbon paste electrode with a 3-n-propyl-4-picolinium chloride silsesquioxane polymer for the detection of HQ isomers. ${ }^{(16)}$ In another report, Han et al. employed an electrochemically reduced hybrid material, ER(GO-TT-CNT), that was prepared by in situ polymerization of graphene oxide (GO), a multiwalled carbon nanotube (CNT), and terthiophene (TT) for the detection of HQ in a pH range of 4.04-9.01.(17)

In one of the earlier reports, Kumar et al. used $\mathrm{ZnO}$ nanoparticles to fabricate an $\mathrm{HQ}$ chemical sensor on a modified glassy carbon electrode. ${ }^{(18)}$ More recently, an efficient and robust $\mathrm{HQ}$ chemical sensor that utilizes Ce-doped $\mathrm{ZnO}$ nanorods as effective electron mediators for the fabrication of an efficient HQ chemical sensor has been reported. ${ }^{(19)}$ Zhang et al. reported a HQ biosensor using modified core-shell magnetic nanoparticles supported on carbon paste. ${ }^{(20)}$ Laccase was covalently immobilized on magnetic nanoparticles by glutaraldehyde, which was modified with amino groups on its surface. ${ }^{(20)}$ In research article published by Li et al. a novel biosensor fabricated on a composite of ZnO-loaded carbon nanofibers, lacasse and Nafion, which shows high efficiency electrocatalysis towards HQ, is reported.(21) However, no report on a perovskite material for HQ detection is found in the literature.

With the above background, a novel HQ sensor is developed using a bimetallic oxide material synthesized through green chemistry. The synthesized material was used as a working electrode of a screen-printed electrode (SPE) and the electrochemical sensing characteristics were determined using a potentiostat from 1 to $9 \mathrm{mM}$ of $\mathrm{HQ}$ at room temperature.

\section{Materials and Methods}

\subsection{Material synthesis}

Hydrous nickel chloride, stannous chloride and organic solvents were purchased from Sigma-Aldrich and purified before use. For all the reactions, deionized water (DI, Millipore, $18 \mathrm{M} \Omega \cdot \mathrm{cm}$ ) was used. The composite $\mathrm{NiSnO}_{3}$ powder was synthesized using chemie douce. In a typical reaction, $11.22 \mathrm{mM}$ of $\mathrm{NiCl}_{2} \cdot 6 \mathrm{H}_{2} \mathrm{O}(2.67 \mathrm{~g})$ and $27.86 \mathrm{mM}$ of $\mathrm{KOH}(1.56 \mathrm{~g})$ were mixed in $15 \mathrm{ml}$ DI water and kept at $80{ }^{\circ} \mathrm{C}$ for $6 \mathrm{~h}$ with continuous stirring. A change in colour from dark green to light green occurred at $\mathrm{pH}=10.5$. The flask was then cooled to room temperature followed by the addition of $11.42 \mathrm{mM}$ $\mathrm{SnCl}_{2} \cdot 2 \mathrm{H}_{2} \mathrm{O}(2.58 \mathrm{~g})$. The entire mixture was then refluxed for $6 \mathrm{~h}$ with stirring at $80{ }^{\circ} \mathrm{C}$. During the reaction, $\mathrm{pH}$ was measured as 7.5 , the refluxing was stopped and the solution was filtered after cooling at room temperature. The reaction resulted in a dark green powder that was obtained after several washing steps with DI water. The synthesized powder was finally calcined at $1000{ }^{\circ} \mathrm{C}$ and then used for sensor development. The proposed method is named as green synthesis mainly due to the low temperature process using an aqueous system resulting in an environmental friendly synthesis. This method is similar to other material synthesis methods such as that reported by Sidorak et al. for the synthesis of cadmium stannates via thermal co-precipitation. ${ }^{(22)}$ 
A scanning electron microscopy (SEM) image was taken using a Hitachi S520 scanning electron microscope. A Philips Tecnai $\mathrm{G}^{2}$ FEI F12 transmission electron microscope was used to obtain a transmission electron microscopy (TEM) image by operation at $80-100 \mathrm{kV}$, and a selected area electron diffraction (SAED) pattern was obtained under similar conditions. The multipoint Brunauer, Emmett, and Teller (BET) technique was used to obtain surface area on a QuadraSorb Station 3 under nitrogen atmosphere after degassing the sample at $200{ }^{\circ} \mathrm{C}$ for $1 \mathrm{~h}$. Dynamic light scattering (DLS) and laser Doppler velocimetry were used for the estimation of the zeta potential in a solution by using Malvern Instruments Zetasizer Nano-ZS instruments. A Fourier transform infrared (FTIR) spectrum was recorded in the range of $4000-400 \mathrm{~cm}^{-1}$ with $\mathrm{KBr}$ pellets in a Perkin Elmer GX spectrometer. An X-ray diffraction (XRD) spectrum was obtained in the reflection mode with $\operatorname{CuK}_{\alpha}(\lambda=1.5406 \AA)$ radiation in the Bragg angle range from 5 to 50 degrees on a Siemens D5000 X-ray diffractometer by continuous diffraction. Thermal analysis was carried out by heating the sample at a rate of $10{ }^{\circ} \mathrm{C} / \mathrm{min}$ from ambient to $1000{ }^{\circ} \mathrm{C}$ in dry air using a Mettler Toledo star analyser.

\subsection{Sensor fabrication and characterization}

Thick films of the synthesized powder were screen-printed over $4 \mathrm{~mm}$ diameter on the working electrode of the SPE, following the procedure reported elsewhere. ${ }^{(23)}$ Goldcoated copper was used as the reference and counter electrodes. The printed films were dried in an oven at $60{ }^{\circ} \mathrm{C}$ and used for sensor characterization. An IVIUM potentiostat/ galvanostat was used to understand the redox reaction of the synthesized material by obtaining a cyclic voltammogram (CV) for a potential cycled from -1.5 to $+1.5 \mathrm{~V}$ with a fixed scan rate of $50 \mathrm{mV} / \mathrm{s}$. To understand the charge transfer kinetics, the scan rate was varied (20 to $80 \mathrm{mV} / \mathrm{s})$ to obtain $\mathrm{CV}$ curves at a particular HQ concentration $(5 \mathrm{mM})$. Different concentrations of HQ were prepared from 1 to $9 \mathrm{mM}$ in phosphate buffer solution ( $\mathrm{pH}=7.4,0.1 \mathrm{M})$. All the measurements were carried out at room temperature. Before measurement, the HQ solution was purged with argon gas for $30 \mathrm{~min}$.

\section{Results}

\subsection{Material characterization}

Morphological observation of the synthesized powder was carried out by SEM and the corresponding low magnification image is shown in Fig. 1(a), where agglomerated particles forming lumps are seen along with tiny particles, which are about 100-200 nm in size. The agglomeration was due to the calcination of the powder at $1000{ }^{\circ} \mathrm{C}$. To further confirm the size and shape of particles, TEM was used for obtaining information about the morphology and crystallinity of the powder and the image is shown in Fig. 1(b), which confirms the SEM observation and clearly shows tiny particles of about 10 $20 \mathrm{~nm}$ size. SAED shows the presence of distinct and good diffraction rings at different planes, which supports the presence of a polycrystalline structure. The difference in size can be due to the measurement method as SEM constructs an image based on the scattered electron profile, while TEM gives an electron-transmission-based image. 


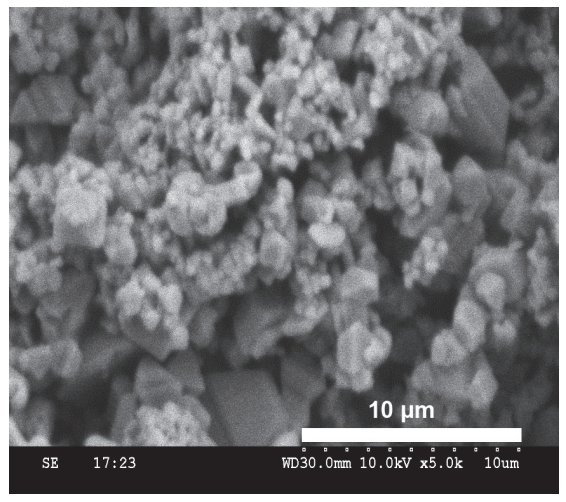

(a)

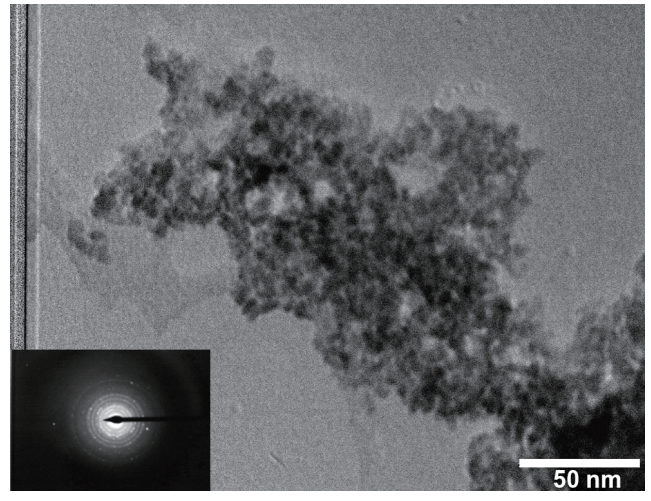

(b)

Fig. 1 (a) SEM image of the synthesized powder and (b) TEM image of the synthesized powder with SAED pattern.

The BET surface area, measured with $\mathrm{N}_{2}$ adsorption, is found to be $\sim 3.95 \mathrm{~m}^{2} / \mathrm{g}$. The zeta measurement was carried out to understand the dispersibility of the synthesized powder. A zeta potential of $-31.5 \mathrm{mV}$ is noted from the measurement indicating that the synthesized particles can give fairly uniform dispersion stability and are less prone to coagulation.

The FTIR spectrum of the synthesized powder is shown in Fig. 2(a), where the presence of two absorption bands is seen at 674 and $547 \mathrm{~cm}^{-1}$ corresponding to the stretching vibrations of $\mathrm{Ni}-\mathrm{O}$ and $\mathrm{Sn}-\mathrm{O}$ bonds, respectively. Asymmetric and symmetric absorption bands due to $\mathrm{Sn}-\mathrm{O}-\mathrm{Ni}$ are observed at 1384 and $944 \mathrm{~cm}^{-1}$, respectively. The occurrence of bands at 3375 and $1627 \mathrm{~cm}^{-1}$ is due to the vibration and deformation frequencies, respectively, of the $\mathrm{OH}$ group since the synthesis was water-based.(24)

The XRD pattern of the synthesized powder is shown in Fig. 2(b), where a mixed phase is observed to contain peaks related to the $\mathrm{NiSnO}_{3}$ phase (JCPDS-28-0711) and $\mathrm{Sn}_{2} \mathrm{O}_{3}$ phase (JCPDS-25-1259). The sharp diffraction peaks indicate the crystalline nature of the synthesized powder. The average particle size calculated using the Scherer formula is about $82 \mathrm{~nm}$, which is close to that observed from SEM images [Fig. 1(a)].

Furthermore, the thermal analysis was carried out to understand the changes in phase purity after the removal of surface impurities with annealing temperature, and the corresponding thermogram is shown in Fig. 2(c). The initial weight loss seen as the first step is due to the removal of unbound water at $100-130{ }^{\circ} \mathrm{C}$ followed by the removal of volatile materials from the particle surface at $130-250{ }^{\circ} \mathrm{C}$, and this finally leads to the formation of crystalline $\mathrm{NiSnO}_{3}$ nanoparticles with phase purity at $545^{\circ} \mathrm{C}$ as illustrated by the presence of an endothermic peak. ${ }^{(25,26)}$ 


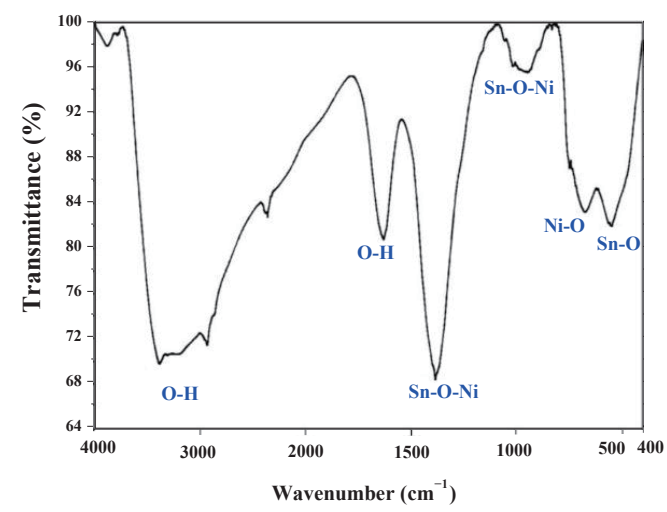

(a)

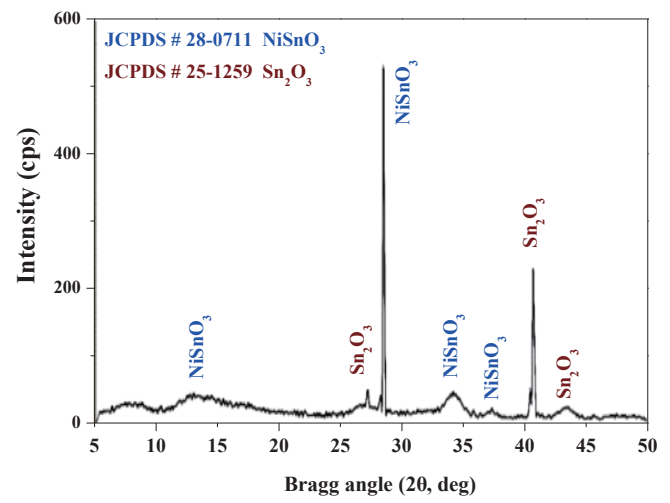

(b)

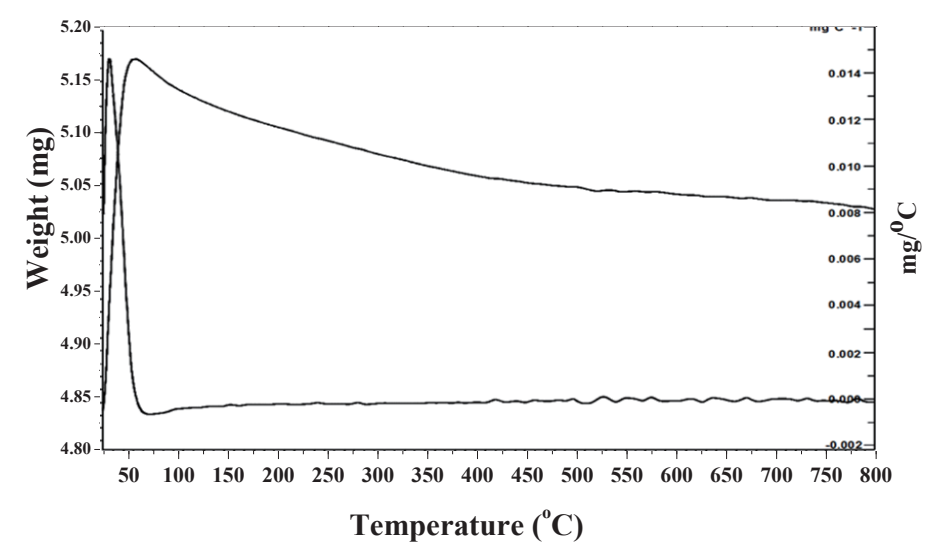

(c)

Fig. 2 (Color online) (a) FTIR spectrum of the synthesized powder, (b) XRD pattern of the synthesized powder, and (c) thermogravimetric data of the synthesized powder.

\subsection{HQ sensing characteristics}

The HQ sensing characteristics are determined through electrochemical characterization, thereby obtaining the $\mathrm{CV}$ in the voltage range of -1.5 to $1.5 \mathrm{~V}$ with a scan rate of $50 \mathrm{mV} / \mathrm{s}$, and are shown in Fig. 3. For this, films of the powder were used as the working electrode of a SPE. Figure 3(a) shows typical CV curves with anodic and cathodic peaks of $\mathrm{NiSnO}_{3}$ powder at different HQ concentrations (0-9 mM). In the CV of PBS, an oxidation peak is seen at $0.4 \mathrm{~V}$ and a reduction peak is seen at 0.04 $\mathrm{V}$, which may be due to the material itself being perovskite in nature. When the CV curve was obtained with HQ, the effect is seen as the appearance of a new redox peak 


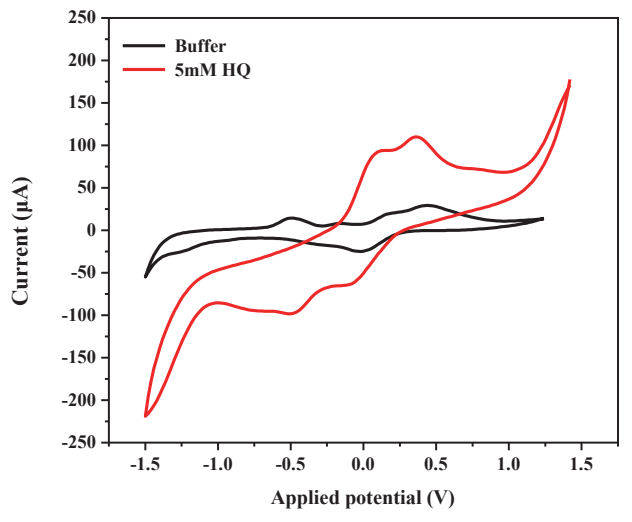

(a)

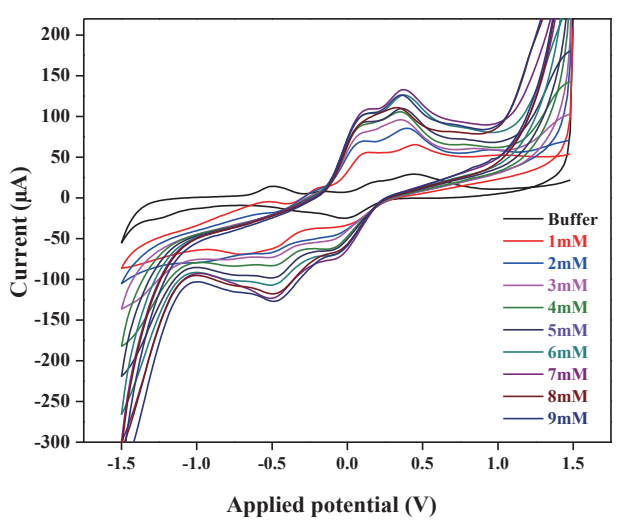

(b)

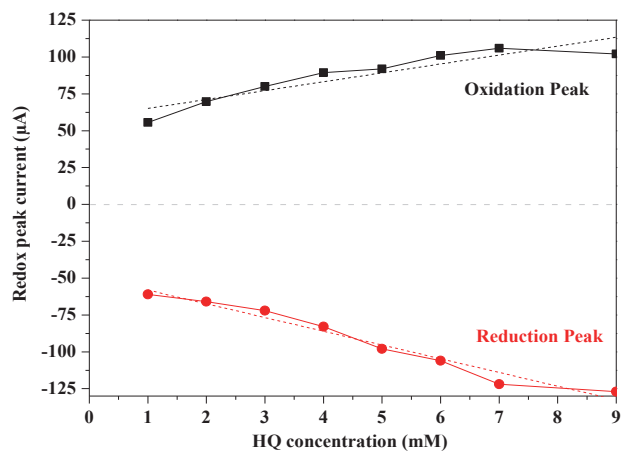

(c)

Fig. 3 (Color online) (a) Typical CV of developed sensor when tested for buffer and $5 \mathrm{mM}$ of $\mathrm{HQ}$ in buffer obtained with applied voltage range of -1.5 to $1.5 \mathrm{~V}$ at a scan rate of $50 \mathrm{mV} / \mathrm{s}$. (b) $\mathrm{CV}$ curves as a function of HQ concentration from 0 to $9 \mathrm{mM}$ of $\mathrm{HQ}$. (c) Variation in the redox peak currents with different HQ concentrations. The dotted line shows the linear fit data.

at 0.1 and $-0.48 \mathrm{~V}$ in addition to those observed in PBS. The redox peaks are found to be separated with a separation potential $\left(\Delta E_{\mathrm{p}}\right)$ of $0.58 \mathrm{~V}$. It is also noted that the redox peak current ratio is greater than unity indicating a reversible process of HQ interaction with $\mathrm{NiSnO}_{3} \cdot{ }^{(18)}$ A variation in the $\mathrm{CV}$ envelope can be seen in Fig. 3(b) with increasing $\mathrm{HQ}$ concentration from 0 to $9 \mathrm{mM}$ of $\mathrm{HQ}$ in buffer solution $(0.1 \mathrm{M}, \mathrm{pH}=7.1)$. Figure 3 (c) shows the variation in the redox peak current at different HQ concentrations. A systematic increase in the peak currents is observed with increasing HQ concentration. The results were further analysed by plotting the oxidation and reduction peak currents with HQ concentration, where increasing oxidation current and decreasing reduction current are observed with increasing HQ concentration. It is noted that the sensor is 
highly sensitive up to $7 \mathrm{mM}$ of $\mathrm{HQ}$ and shows a slight deterioration in the performance at a higher concentration. It can be considered that the sensor can sensitively detect the presence of HQ within the safe limit designated as $5 \mathrm{mM} \cdot \cdot^{(1,2,13,18)}$ The sensitivity estimated from this curve is $6.03 \mu \mathrm{A} / \mathrm{mM}\left(46.38 \mu \mathrm{A} / \mathrm{mMcm}^{-2}\right)$ in the case of oxidation current and $-9.31 \mu \mathrm{A} / \mathrm{mM}\left(71.61 \mu \mathrm{A} / \mathrm{mMcm}^{-2}\right)$ in the case of reduction current values with a linearity over $90 \%$, as estimated from the linear fit equation.

From the literature survey, it is noted that $\mathrm{NiSnO}_{3}$ has not been used for HQ sensing purposes, however, the sensor performance is compared with some of the reported work and listed in Table 1. Most of the sensors reported are based on graphene or CNTs, with varying sensor parameters, which makes it difficult to compare the material superiority. However, it is clear that the developed sensor has comparable performance with reported sensors in the literature. ${ }^{(28-35)}$

\section{Discussion}

To understand the charge transfer characteristics of the sensor material, a scan rate study was carried out and CVs were obtained at various scan rates $(20,30,40,50,60$, 70,80 , and $90 \mathrm{mV} / \mathrm{s}$ ) in a $5 \mathrm{mM}$ HQ solution. A systematic change in the oxidation/ reduction peak current is observed with increasing scan rate as shown in Fig. 4(a), which shows a linear variation (increasing with consistency) with the scan rate indicating that the redox reaction at the working electrode is a surface-controlled process since the adsorption-controlled current contributes majorly to the peak current. To understand the diffusion-controlled process, the peak currents were plotted as square root of the scan rate $\left(v^{1 / 2}\right)$, which exhibited a progressive response (linearly increasing with scan rate) indicating a diffusion-controlled process due to a fast electron transfer mechanism, as can be seen in Fig. 4(b). Furthermore, the variation in the redox peak current with natural log of scan rate $(\ln v)$ shows a linear relationship $\left[I_{\text {(oxidation) }}=-57.85+43.37(\ln v)\right.$ with $R^{2}=0.9783$,

Table 1

Comparison with reported hydroquinone sensors fabricated using various materials as mediators.

\begin{tabular}{|c|c|c|}
\hline Materials & $\begin{array}{c}\text { Sensitivity } \\
\left(\mu \mathrm{A} \mu \mathrm{M}^{-1} \mathrm{~cm}^{-2}\right)\end{array}$ & $\begin{array}{l}\text { Limit of detection } \\
\text { (M) }\end{array}$ \\
\hline Dinuclear copper (II) complex in carbon paste ${ }^{(27)}$ & - & $3.0 \times 10^{-7}$ \\
\hline Graphene modified GCE ${ }^{(28)}$ & - & $1.5 \times 10^{-8}$ \\
\hline L-Cys/Au SAMs ${ }^{(29)}$ & - & $4.0 \times 10^{-7}$ \\
\hline Poly(thionine) modified $\mathrm{GCE}^{(30)}$ & 1.8 & $30 \times 10^{-3}$ \\
\hline MWCNT modified GCE ${ }^{(31)}$ & - & $75 \times 10^{-4}$ \\
\hline Platinum and graphene modified $\mathrm{GCE}^{(32)}$ & 1.38 & $1.2 \times 10^{-5}$ \\
\hline Reduced graphene oxide and MWCNT modified $\mathrm{GCE}^{(33)}$ & - & $2.6 \times 10^{-6}$ \\
\hline Graphene modified $\mathrm{GCE}^{(34)}$ & 1.38 & $12 \times 10^{-6}$ \\
\hline $\mathrm{NiSnO}_{3}$ nanopowder (present work) & $46.38 \times 10^{-3}$ & $78 \times 10^{-5}$ \\
\hline
\end{tabular}




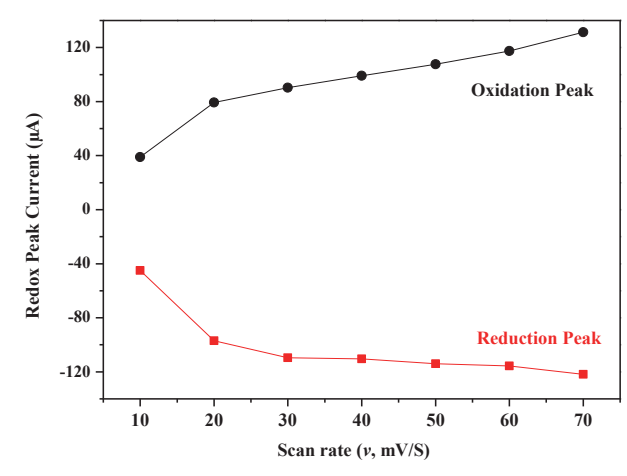

(a)

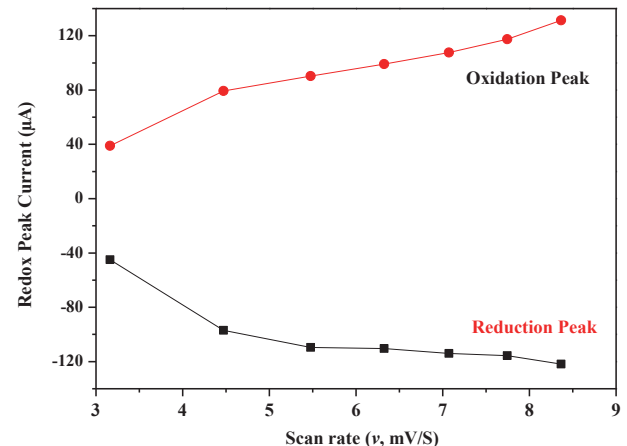

(b)

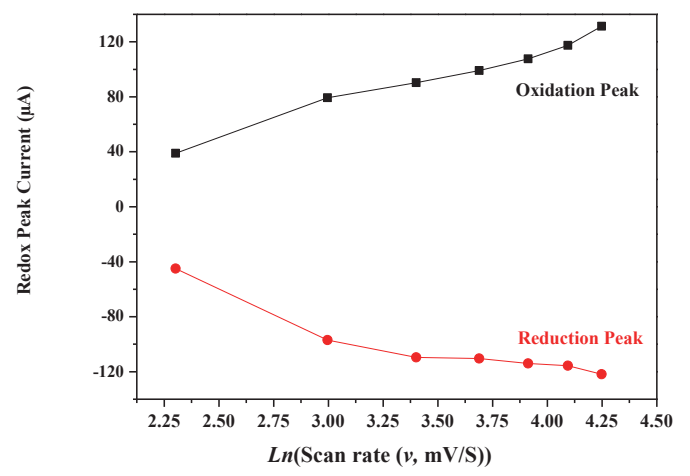

(c)

Fig. 4 (Color online) (a) Variation in the redox peak current with different scan rates at $5 \mathrm{mM}$ of $\mathrm{HQ}$, (b) variation in the redox peak current with square root of scan rate at $5 \mathrm{mM}$ of $\mathrm{HQ}$, and (c) variation in the redox peak current with natural $\log$ of scan rate at $5 \mathrm{mM}$ of HQ.

estimated from the linear fitting of the curve] between peak currents and scan rate $(\ln v)$, as shown in Fig. 4(c), indicating that the electrode reaction is diffusion-controlled. To confirm the diffusion-controlled process, a logarithmic plot of peak current and scan rate was plotted and the plot shows a linear relationship with a linearity of $92 \%$ and a slope of 0.56 , which is close to the theoretical value of 0.5 , confirming that the electrode reaction is a diffusion-controlled process. ${ }^{(13)}$

As seen from XRD data, the material is a mixed phase of $\mathrm{NiSnO}_{3}$ and $\mathrm{Sn}_{2} \mathrm{O}_{3}$, which can be easily oxidized by the adsorption of atmospheric oxygen. The metal ions on the surface can further facilitate this process and provide better sensing characteristics. ${ }^{(27)}$ The adsorbed oxygen will provide an oxygen species that will help in the oxidation/reduction of the material upon interaction with HQ. The performance of the developed sensor is comparable to that of the reported sensors, ${ }^{(16-21)}$ presenting a possibility of exploring new materials for sensing applications. 


\section{Conclusions}

Green synthesis was used to synthesize the perovskite structure of nickel and tin oxide as $\mathrm{NiSnO}_{3}$. The synthesis resulted in tiny nanoparticles of about $10-20 \mathrm{~nm}$ size with polycrystalline nature. FTIR and thermogravimetric studies were conducted to confirm the functional properties and phase purity of the synthesized nanoparticles. An electrochemical sensor in the form of SPE for HQ detection properties was investigated from $0-9 \mathrm{mM}$ of HQ in buffer solution. The developed sensor exhibited a linear response up to $7 \mathrm{mM}$ of HQ, which is above the safe limit of HQ usage. The charge transfer studies indicated that the reaction at the working electrode is a diffusioncontrolled process. It is realized that the synthesized material can be used as a promising material for various electrochemical sensing applications.

\section{Acknowledgements}

The authors would like to extend their sincere appreciation to the Deanship of Scientific Research at King Saud University for funding this research group number RG1435-052. The authors acknowledge the measurement support received from the Central Instrumentation Facility of Jamia Millia Islamia, New Delhi, India.

\section{References}

1 D. Neagu, G. Tsekouras, D. N. Miller, H. Ménard and J. T. S. Irvine; Nat. Chem. 5 (2013) 916.

2 J. Borges, C. Fonseca, N.P. Barradas, E. Alves, T. Girardeau, F. Paumier, F. Vaz and L. Marques: Electrochim. Acta 106 (2013) 23.

3 T. Athar: Emerging Nanotechnologies for Manufacturing, eds. W. Ahmed and J. Mark Jackson (William Andrew (Elsevier), MA, USA, 2015, 2nd ed.) Chap. 14, p. 343.

4 S. Das and V. Jayaraman: Prog. Mater. Sci. 66 (2014) 112.

5 M. José-Yacamán and R. Mehl: Metall. Mater. Trans. A 29 (1998) 713.

6 G. George and S. Anandhan: Mater. Sci. Semicond. Process. 32 (2015) 40.

7 X. Jia, H. Fan, X. Lou and J. Xu: Appl. Phys. A 94 (2009) 837.

8 X. Li, Y. Zhong, M. Cai, M. P. Balogh, D. Wang, Y. Zhang, R. Li and X. Sun: Electrochim. Acta 89 (2013) 387.

9 Rajesh, T Ahuja and D Kumar: Sens. Actuators, B 136 (2009) 275.

10 S. B. Khan, M. M. Rahman, K. Akhtar and A. M. Asiri: PLoS ONE 9 (2014) e109423.

11 J. L. O’Donoghue: J. Cosmet. Dermatol. 5 (2006) 196.

12 F. J. Enguita and A. L. Leitão: Biomed. Res. Int. 2013 (2013) Article ID 542168.

13 S. Hu, Y. Wang, X. Wang, L. Xu, J. Xiang and W. Sun: Sens. Actuators, B 168 (2012) 27.

14 J. Zou, J. Ma, Y. Zhang, L. Huang and Q. Wan: J. Chem. Technol. Biotechnol. 89 (2014) 259.

15 H. Du, J. Ye, J. Zhang, X. Huang and C. Yu: J. Electroanal. Chem. 650 (2011) 209.

16 P. S. da Silva, B. C. Gasparini, H. A. Magosso and A. Spinelli: J. Braz. Chem. Soc. 24 (2013) 695.

17 H. S. Han, J. M. You, H. Seol, H. Jeong and S. Jeon: Sens. Actuators, B 194 (2014) 460.

18 R. Kumar, D. Rana, A. Umar, P. Sharma, S. Chauhan and M. S. Chauhan: Sens. Lett. 12 (2014) 1273. 
19 G. N. Dar, A. Umar, S. A. Zaidi, Ahmed A. Ibrahim, M. Abaker, S. Baskoutas and M. S. AlAssiri: Sens. Actuators, B 173 (2012) 72.

20 Y. Zhang, G. M. Zeng, L. Tang, D. L. Huang, X. Y. Jiang and Y. N. Chen: Biosens. Bioelectron. 22 (2007) 2121.

21 D. Li, J. Yang, J. Zhou, Q. Wei and F. Huang: RSC Adv. 4 (2014) 61831.

22 A. V. Sidorak, V. V. Ivanov and A. A. Shubin: Mater. Sci. Appl. 2 (2011) 1219.

23 Z. A. Ansari, S. Khalid, A. A. Khan, H. Fouad and S. G. Ansari: Sens. Lett. 12 (2014) 1495.

24 G. Soacrates: Infrared and Raman Characteristic Group Frequencies: Tables and Charts (John Wiley \& Sons Ltd., England, 2001) pp. 50, 80.

25 P. Gabbott (ed.): Principles and Applications of Thermal Analysis (Wiley-Blackwell Publishing, UK, 2008) p. 410.

26 M. S. Niasaria, N. Mir and F. Davar: Polyhedron 28 (2009) 1111.

27 W. Yude, S. Xiaodan, L. Yanfeng, Z. Zhenlai and W. Xinghui: Solid-State Electron. 44 (2000) 2009.

28 I. R. W. Z. de Oliveira, R. E. M. d. Osorio, A. Neves and I. C. Vieira: Sens. Actuators, B 122 (2007) 89.

29 B. Kong, T. Yin, X. Liu and W. Wei: Anal. Lett. 40 (2007) 2141.

30 H. Du, J. Ye, J. Zhang, X. Huang and C. Yu: J. Electroanal. Chem. 650 (2011) 209.

31 L. F. Fan, X. Q. Wu, M. D. Guo and Y. T. Gao: Electrochim. Acta 52 (2007) 3654.

32 A. J. S. Ahammad, M. M. Rahman, G. R. Xu, S. Kim and J. J. Lee: Electrochim. Acta 56 (2011) 5266.

33 S. Wang and D. Du: Sensors 2 (2002) 41.

34 X. Cao, X. Cai, Q. Feng, S. Jia and N. Wang: Anal. Chim. Acta 752 (2012) 101.

35 J. Li, C. Y. Liu and C. Cheng: Electrochim. Acta 56 (2011) 2712. 